\title{
Associations of individual and family eating patterns during childhood and early adolescence: a multicentre European study of associated eating disorder factors
}

\author{
Isabel Krug ${ }^{1,2}$, Janet Treasure ${ }^{3,4}$, Marija Anderluh ${ }^{5}$, Laura Bellodi ${ }^{6}$, Elena Cellini ${ }^{7}$, David Collier ${ }^{4}$, \\ Milena di Bernardo ${ }^{7}$, Roser Granero ${ }^{8}$, Andreas Karwautz ${ }^{9}$, Benedetta Nacmias ${ }^{7}$, Eva Penelo ${ }^{8}$, Valdo Ricca ${ }^{7}$, \\ Sandro Sorbi ${ }^{7}$, Kate Tchanturia ${ }^{4}$, Gudrun Wagner ${ }^{9}$ and Fernando Fernández-Aranda ${ }^{1,2}{ }^{2}$ \\ ${ }^{1}$ Department of Psychiatry, University Hospital of Bellvitge, c/ Feixa Llarga s/n, 08907-Barcelona, Spain \\ ${ }^{2}$ Ciber Fisiopatologia de la Obesidad y Nutricion (CIBERobn), Instituto Salud Carlos III, 08907 Barcelona, Spain \\ ${ }^{3}$ Department of Psychiatry, 5th Floor, Thomas Guy House, Guy's Hospital, London SE1 9RT, UK \\ ${ }^{4}$ South London and Maudsley Eating Disorders, Institute of Psychiatry/King's College, London SE 58AF, UK \\ ${ }^{5}$ University Children's Hospital, University Medical Centre Ljubljana, Vrazov trg 1, 1000 Ljubljana, Slovenia \\ ${ }^{6}$ Department of Neuropsychiatric Sciences (DSNP), Fondazione Centro S. Raffaele del Monte Tabor, Via Olgettina 60, 20132 \\ Milan, Italy \\ ${ }^{7}$ Department of Neurology and Psychiatric Sciences, University of Florence, Viale Morgagni 85I-50134, Florence, Italy \\ ${ }^{8}$ Departament de Psicobiologia i Metodologia, Universitat Autònoma de Barcelona, Facultat de Psicologia, Edifici B (Campus \\ Bellaterra), 08193 Bellaterra (Cerdanyola del Valles), Spain \\ ${ }^{9}$ Department of Child and Adolescent Psychiatry, Medical University of Vienna, Währinger Gürtel 18-20, 1090 Vienna, Austria \\ (Received 12 March 2008 - Revised 27 May 2008 - Accepted 17 June 2008 - First published online 28 August 2008)
}

\begin{abstract}
The objective of this study was to examine whether there is an association between individual and family eating patterns during childhood and early adolescence and the likelihood of developing a subsequent eating disorder (ED). A total of 1664 participants took part in the study. The ED cases $(n$ 879) were referred for assessment and treatment to specialized ED units in five different European countries and were compared to a control group of healthy individuals ( $n$ 785). Participants completed the Early Eating Environmental Subscale of the Cross-Cultural (Environmental) Questionnaire, a retrospective measure, which has been developed as part of a European multicentre trial in order to detect dimensions associated with ED in different countries. In the control group, also the General Health Questionnaire-28 (GHQ-28), the semi-structured clinical interview (SCID-I) and the Eating Attitudes Test (EAT-26) were used. Five individually Categorical Principal Components Analysis (CatPCA) procedures were adjusted, one for each theoretically expected factor. Logistic regression analyses indicated that the domains with the strongest effects from the CatPCA scores in the total sample were: food used as individualization, and control and rules about food. On the other hand, healthy eating was negatively related to a subsequent ED. When differences between countries were assessed, results indicated that the pattern of associated ED factors did vary between countries. There was very little difference in early eating behaviour on the subtypes of ED. These findings suggest that the fragmentation of meals within the family and an excessive importance given to food by the individual and the family are linked to the later development of an ED.
\end{abstract}

Eating disorders: Eating problems: Eating behaviour: Adolescence: Anorexia nervosa: Bulimia nervosa: Childhood: Family

A recent systematic review of the risk factor literature suggested that early eating and gastrointestinal difficulties may be developmental factors of relevance for eating disorders $(\mathrm{ED})^{(1)}$. However, research into early patterns of eating behaviour has been limited as the size of prospective studies has rarely been large enough to have sufficient power to be confident in the findings for ED and the detail about the form of early eating risk may be insufficient ${ }^{(2-7)}$.

\section{Individual eating patterns during childhood and early} adolescence

Research has shown that eating behaviours in childhood do appear to be linked to the later development of $\mathrm{ED}^{(8-10)}$. The two longitudinal studies addressing this topic reported that early problematic eating patterns are associated with subsequent unhealthy eating behaviours ${ }^{(11,12)}$ and that

Abbreviations: AN, anorexia nervosa; AUC, area under the receiver operator curve; BN, bulimia nervosa; CatPCA, categorical principal components analysis; EAT-26, Eating Attitudes Test; ED, eating disorder; EDNOS, eating disorders not otherwise specified; GHQ-28, General Health Questionnaire-28; SCID-I, semi-structured clinical interview.

* Corresponding author: Dr Fernando Fernández-Aranda, fax +34 93 2607658, email ffernandez@csub.scs.es 
eating conflicts and struggles around meals increased the risk of anorexia nervosa (AN) later in life, whereas eating too little protected against the development of bulimia nervosa $(\mathrm{BN})^{(13)}$.

Several retrospective studies on $\mathrm{AN}$ and $\mathrm{BN}$ have also addressed this issue $\mathrm{e}^{(10,14)}$. Even though this type of design has limitations, it can nevertheless be used to explore and define potential risk factors. In relation to $\mathrm{AN}$, studies have indicated that compared to controls, mothers of individuals with AN reported more extreme feeding problems and severe gastrointestinal problems of their children during infancy ${ }^{(14,15)}$. Early childhood eating and problems for BN have been contradictory. While one study ${ }^{(10)}$ of sister discordant for ED found that BN individuals were more often overweight with less picky eating and eating more quickly during infancy than their healthy siblings, Marchi \& Cohen ${ }^{(12)}$ revealed that pica, early digestive difficulties and weight-loss attempts were related to subsequent bulimic behaviours. Further studies are therefore needed to clarify these inconsistencies.

\section{Family eating patterns during childhood and early} adolescence

Research has shown that children's attitudes towards food and children's evaluation of satiety and appetite are influenced by their parents and their family environment ${ }^{(16,17)}$. Parents control the food environment and operate as models for eating and food-related behaviours. In relation to $\mathrm{ED}$, research on the effects of familial factors on problematic eating behaviours in childhood has not frequently been examined ${ }^{(18)}$. The few findings in this field have been contradictory with some studies indicating that a restrictive feeding style by parents was related to the development of overweight in their children $^{(19)}$. In contrast, parental control over eating was related to eating fewer meals and snacking less frequently ${ }^{(20)}$. Moreover, critical comments about eating, weight and shape from family members have been found to be associated with bulimic forms of $\mathrm{ED}^{(21,22)}$. However, research has not yet addressed whether distinct parental attitudes to food are associated with different ED subtypes.

\section{Cultural influences on eating disorders}

Cultural differences in individual and family eating styles and whether these might impact on the development of ED has rarely been addressed. It is, nevertheless, generally accepted that food preferences are culturally learned and that different countries have their own distinct diets and attitudes towards food $^{(23-25)}$. Sociocultural factors are important contributing factors in the development of $\mathrm{ED}^{(1,26,27)}$. This is particularly relevant for the bulimic disorders which suddenly emerged in cohorts born after 1950 in the USA and Western Europe ${ }^{(26)}$. In spite of this, research in this area has predominantly assessed ethnic minority groups residing in the $\mathrm{UK}^{(28)}$ or the USA ${ }^{(29-31)}$. There have been fewer comparisons of environmental risk factors between countries and even fewer across Europe ${ }^{(32-34)}$.

To summarize, the confidence that can be placed in findings from the literature on early risk factors for ED is limited. The few studies that have addressed this topic have yielded conflicting results. The scope of possible risk factors is limited as are the outcomes used. Nonetheless, there is some evidence to suggest that the development of unhealthy eating attitudes and behaviours in early childhood and parental influence upon children's eating styles can be part of the developmental trajectory into ED.

\section{Aims of the study}

The overall aim of the present study was to recruit a large sample of patients and healthy controls from the range of environments within Europe. Our specific objectives were: (1) to examine in more detail some of the early patterns of eating behaviour which may be associated with the development of a subsequent ED; (2) to assess whether there were differences across countries in these early putative associated eating behaviours; (3) to evaluate whether there were differences among the distinct ED sub-diagnoses in early individual and family eating behaviour; and (4) whether these differed between the different countries. We hypothesized that unhealthy individual and family eating styles in childhood and early adolescence would be related to an ED later in life and that ED sub-diagnoses and countries would differ in these associated eating behaviours.

\section{Methods \\ Participants}

The present study employed a case-control design. Six centres from five different European countries (two for Italy) participated in the current study: the University Hospital of Bellvitge, Barcelona, Spain (ED, $n$ 262, control, $n$ 160); the Medical University of Vienna; Department of Child and Adolescent Psychiatry (ED, $n$ 94, control, $n$ 59); the Eating Disorders Research Unit, Institute of Psychiatry, London, UK (ED, $n$ 319, control, $n$ 184); the Department of Neurology and Psychiatric Services, University of Florence, Italy (ED, $n$ 50, control, $n$ 50); the Department of Psychiatry, Fondazione Centro del Monte Tabor, Milan, Italy (ED, $n$ 93, control, $n$ 101) and the University Psychiatric Hospital, University of Ljubljana, Slovenia (ED, $n$ 61, control, $n$ 231). Entry into the study was between March 2001 and September 2002. The total sample comprised 1664 participants, 879 ED patients $(42.2 \%$ with $\mathrm{AN}, 32.2 \%$ with $\mathrm{BN}$ and $25.6 \%$ with eating disorders not otherwise specified (EDNOS)) and 785 healthy controls. Considering the ED sub-diagnoses, we found that $52.8 \%$ of the AN patients were diagnosed with the restrictive subtype and $47.2 \%$ with the bulimic-purgative subtype. This distribution was statistically equal for countries $(P=0.071)$. Due to the limited sample size of non-purging BN individuals a distinction between purging and non-purging $\mathrm{BN}$ subtypes could not be made.

Most ED participants were ascertained from the participating clinical sites and the remaining individuals were collected from different therapeutic institutions for ED, selfhelp groups, announcements in the mass media and on ED conferences. Participants were ill at assessment and were diagnosed according to DSM-IV-R ${ }^{(35)}$ criteria, using a semi-structured clinical interview (SCID-I) ${ }^{(36)}$ or EATAET (M Anderluh et al., unpublished results; only used for Austria and the UK), carried out by experienced psychologists and psychiatrists. 
The interviewers were trained in the administration of these instruments although formal inter-rater reliability was not computed for the present study. The exclusion criteria for the ED patients in the present study were: (1) individuals who had not completed a diagnostic assessment; (2) younger than 16 years old; (3) unable to complete the assessment because of cognitive impairment and/or serious medical condition; or (4) current psychotic disorder. For the present analysis, the following ED individuals had to be excluded from an initial sample of 901 patients: (1) patients ( $n$ 18) did not have a diagnosis measured; (2) cognitive impairment ( $n$ 2); (3) comorbid psychotic disorder ( $n$ 2).

Healthy controls ( $n$ 785) with similar demographic features (sex, age and education) to the clinical participants were ascertained from various community sources from the same catchment area. The exclusion criteria for the control group were: (1) younger than 16 years; (2) a lifetime history of ED was assessed by the General Health Questionnaire-28 $8^{(37)}$, the SCID-I ${ }^{(36)}$ according to DSM-IV-R criteria ${ }^{(35)}$ and the Eating Attitudes Test (EAT-26) ${ }^{(38)}$ (total score $>20$ ). From the initial sample of 791 controls, six participants were excluded, who had had a lifetime ED. Ethical approval for the study was obtained from the relevant committees at each site.

The mean age of the total sample was $25 \cdot 3$ (SD $8 \cdot 7$ ) years. The ED patients were significantly older than the controls (ED cohort: mean 26.7 (SD 8.9) years; control group: mean 23.8 (SD 8.2 ); $P=0.001$ ). The gender ratio also differed, the ED group comprised significantly more females than the control group (ED cohort: female 96.4\%; control group: female $91.1 \% ; P=0.001)$. Also more ED patients were employed (ED cohort: employed 54.9\%; control group: employed $39.1 \% ; P=0.001)$ whereas more controls were currently studying (ED cohort: student $47.80 \%$; control group: student $75.32 \% ; P=0.001$ ), which might be attributable to the lower age in the control group. More ED cases had grown up in urban places (ED cohort: urban 68.97\%; control group: urban $56.78 \% ; P=0.001)$. More than half of the sample came from families with one or more sisters $(58.07 \%)$ and had advanced education $(54.53 \%)$. The mean age of onset of the ED was 19.32 (SD 5.05) years and the mean duration of the disorder was 7.11 (SD 5.44) years. The median of previous treatments was 1 (ranging from 0 to 5). Participants reported a weekly average of 4.8 (SD 5.8) binge eating episodes and 7.1 (SD 8.7) vomiting episodes. Their mean BMI at assessment was $20.44($ SD 6.53$) \mathrm{kg} / \mathrm{m}^{2}$.

\section{Assessment}

The Early Eating Environmental Subscale of the CrossCultural (Environmental) Questionnaire. This retrospective self-administered questionnaire has fifty-one items with six subscales. It was developed by an expert group from various European countries in order to detect environmental factors associated with the development of ED (childhood eating patterns, meaning and value of food, family style, independence, parenting, self-development and social ideals of thinness and fitness and activity). The Cross-Cultural (Environmental) Questionnaire was based on the major instruments in the field of ED, which are the Oxford Risk Factor Interview ${ }^{(21-39)}$ and the McKnight Risk Factor Interview ${ }^{(40)}$. The internal consistency of the derived scales fluctuated between good and very good (Cronbach's $\alpha$ coefficients between 0.75 and $0 \cdot 88$ ). The dimensional scores derived from the factor analysis discriminated adequately between patients and controls $(P \leq 0.05)$ and the global diagnostic capacity of the test was found to be satisfactory (area under the receiver operator curve (AUC) $\geqq 0 \cdot 80)^{(42)}$.

The items for the eating section of the questionnaire were developed from a focus group with patients and following a review of the literature with an expert group of clinicians. The items generated by this process were then grouped into themes by three expert clinicians. There were five theoretical domains: food used as individualization, control and rules about food, food utilized as social glue, healthy eating and food deprivation. In the current study, only twenty-six of the twenty-nine items of the food and eating family style subscale were considered. Two items were not included due to the lack of cases with positive answers ('food prepared specially for others' and 'specific attention to healthy eating by others'). Moreover, another item was excluded because it measured current instead of childhood eating patterns ('eat meals together after 12 years'). A detailed description and a copy of this subscale can be found in another recent publication and can be requested from the corresponding author ${ }^{(8)}$. A very high agreement, with an average value of 0.92 (95\% CI $0.89,0.93$ ) was found for all the items.

EATATE Phenotype Interview. The EATATE interview was developed for the European Healthy Eating Project. It is a semi-structured interview, comprising a European adaptation of the Longitudinal Interval Follow-up Evaluation ${ }^{(42)}$ and the Eating Disorders Examination ${ }^{(43)}$. The interview is used to obtain a life-time history of ED symptoms, which are then plotted on a lifeline. This instrument has been validated and has demonstrated good inter-rater reliability in terms of diagnoses $(0.82-1.0)$ and illness history variables (0.80-0.99) (M Anderluh et al., unpublished results).

General Health Questionnaire. The General Health Questionnaire-28 (GHQ-28) ${ }^{(37)}$ is a self-report questionnaire that has been designed to detect and assess individuals with an enlarged probability of a present psychiatric disorder. The GHQ-28 has been studied in various European countries and was found to be a valid and reliable tool ${ }^{(44)}$.

Eating Attitudes Test. The EAT-26 ${ }^{(38)}$ assesses a broad range of symptoms and provides a total score for disturbed eating attitudes and behaviour. This instrument has acceptable criterion related validity with Cronbach's $\alpha$ ranging from 0.82 to 0.89 in a previous study.

\section{Procedure}

Participants were invited to participate in a European multicentre study. All patients were first assessed by board-certified psychologists or psychiatrists using a $2 \mathrm{~h}$ structured diagnostic face-to-face or phone interview to measure ED symptoms and psychopathological traits. ED diagnoses were based on this interview and were consensually derived among members of the clinical team who had participated in the assessment. An information sheet at the start of the questionnaire informed the participants about the purpose of the study and assured confidentiality of the results. 
Furthermore, it was emphasized that participation in the study was completely voluntary and that participants were free to withdraw from the study at any time.

\section{Statistical analysis}

Statistical analysis was carried out with SPSS version 15.0.1 for Windows (SPSS Inc., Chicago, IL, USA). Firstly, through the categorical principal components analysis (CatPCA) procedure, we obtained an empirical reduction of the set of twenty-six variables which make up the early eating subscale included in the analysis. This method is included in the optimal scaling procedures and can be used for uncovering the underlying structure (factors or dimensions) of a large set of variables; therefore it reduces the income of a large set of variables to a reduced number of underlying factors. It is adequate for variables with mixed scaling levels (including nominal levels) and it assumes that the relationship among observed variables is not linear. CatPCA was carried out in the whole European clinical sample (n 879). Five individually CatPCA analyses were adjusted, one for each theoretically expected factor. In each analysis the number of dimensions was fixed a priori at one (one-dimensional solution). First, we obtained and interpreted the factor or component loadings, which represent the correlation coefficients between the original income variables and the new factors that emerged. Next, factor scores of each dimension were obtained for each participant. In the context of principal component analysis, a factor score is a numerical value which indicates a person's relative spacing or standing on the latent emerged factor. In the present study, factor scores were computed including all the items analysed in each model, independently of its factor loading since in this work content is considered more important than reliability ${ }^{(45)}$. The same coefficient matrix was used to calculate factor scores in the control sample.

Factor scores were compared between cases and controls for the total European sample and stratified by countries, with ANOVA procedures adjusted by sex and age. Next, the five empirical factor scores were included as independent variables into logistic and multinomial regressions (ENTER procedure) adjusted by sex and age to examine their predictive accuracy on the presence of an ED diagnosis (present $v$. absent, logistic regressions) and ED subtypes (absent-AN-BN-EDNOS, multinomial regressions). In the logistic models, significant OR higher than 1 indicate that higher factor scores increase the risk of suffering from an ED. In the multinomial models, the reference category was defined for the control group, and consequently significant OR higher than 1 indicate that higher factor scores increase the risk of reporting the concrete ED subtype. The global predictive accuracy of each model was valued with the Nagelkerke's $R^{2}$ coefficient and through the AUC. As it is commonly interpreted, we considered that AUC values between 0.60 and 0.70 are moderate, between 0.70 and 0.80 are good and above 0.80 are excellent. The adjustment was measured through Hosmer-Lemeshow's tests $(P$ values higher than 0.05 are indicative of adequate adjustment). Independent models were constructed for data provided separately by each country and also for the total European sample.

\section{Results}

\section{Results of the categorical principal components analysis} procedure

Table 1 contains the factor loadings of each model obtained through the CatPCA procedures. Factor 1 was related to the theme of valuing food as a method of individualization (food specially prepared for family members), with especially high loadings for items measuring food prepared for father, sibling and the participant herself. Factor 2 was associated with the theme of control and rules about food, with the highest loadings for the items 'parents had strict rules about food', 'food was used as reward' and 'access to food restricted as punishment'. Factor 3 was linked to the employment of food as social glue and obtained the highest loadings for the two items 'patient was included in social meals' and 'number of foods included as a social event'. Factor 4 encompassed healthy eating, and it was especially associated with the parents', siblings' and the individual's own attention to healthy eating and also with restricted access to snacks and fast food. Factor 5 was associated with food deprivation and included the items shortage of basic and luxury food in the family (both with high factor loadings). A careful look at the items suggests that it could also reflect poverty or inadequate funds to buy foods in general or luxury foods specifically. The total variance explained by each factor was

Table 1. Factor loadings in categorical principal components analysis procedure in the European clinical sample $(n 879)$

Component loadings

\begin{tabular}{lr}
\hline Factor 1: Food used as individualization & \\
Food prepared specially for grandparent(s) & 0.02 \\
Food prepared specially for mother & 0.09 \\
Food prepared specially for father & 0.80 \\
Food prepared specially for sibling & 0.83 \\
Food prepared specially for patient & $0 \cdot 81$ \\
Factor 2: Control and rules about food & \\
Parents had strict rules about food & 0.54 \\
Follow parents' rules about food usually & -0.40 \\
Food was usually used as a reward & $0 \cdot 68$ \\
Access to food restricted - punishment & 0.74 \\
Factor 3: Food utilized as social glue & \\
No. of times ate meals together & 0.03 \\
before 12 years & \\
Ate meals at regular/set times of the day & -0.11 \\
No. of family members present at meals & -0.17 \\
Patient was included in social meals & 0.90 \\
No. of meals were included as social event & 0.91 \\
Value placed on food by mother & -0.27 \\
Value placed on food by father & -0.30 \\
Factor 4: Healthy eating & \\
Had first meal of day & -0.04 \\
(breakfast before school) & \\
Mother paid attention to healthy eating & 0.60 \\
Father paid attention to healthy eating & 0.53 \\
Sibling paid attention to healthy eating & 0.47 \\
No. of times ate in fast food restaurants & -0.17 \\
Patients paid attention to healthy eating & 0.56 \\
Ate fatty/sugary snacks usually & -0.48 \\
Access to salty/snacks was restricted & 0.52 \\
Factor 5: Food deprivation & \\
Shortage of basic food in family & 0.82 \\
Shortage of luxury foods in family & 0.82
\end{tabular}


satisfactory, with values ranging from $21.2 \%$ (for factor 4, 'healthy eating') to $39.8 \%$ (for factor 1 , 'food used as individualization'). Reliability based on Cronbach's $\alpha$ values varied from moderate $(0.49$, for factor 5 , 'food deprivation') to high ( 0.76 , for factor 1 , 'food used as individualization'). These results can be valued as adequate considering the reduced number of items contained in the factors.

The comparison of the frequency distribution for ED subtype (AN-BN-EDNOS) obtained some statistical associations. Concretely, food prepared specially for another sibling achieved a higher prevalence for AN patients $(15.7 \%)$ than for $\mathrm{BN}(7.5 \%)$ or $\operatorname{EDNOS}(10.3 \%)$ individuals $(P=0 \cdot 012)$. Considering the number of times that the respondent was included in 'social meals' (once a week or more), AN and EDNOS patients achieved higher percentages (11.5 and $13.6 \%$, respectively) than $\mathrm{BN}$ patients $(4.8 \%)(P=0.006)$. Compared to AN $(11.8 \%)$ and EDNOS $(9.6 \%)$, a higher percentage of BN patients $(17.2 \%)$ had mothers who valued food a lot more than most people $(P=0 \cdot 021)$. This trend was similar when the amount of value placed on food by the father was assessed (BN, 11.5\%; AN, 6.9\%; EDNOS, 5.2\%; $P=0.002)$. Furthermore, results indicated that $25.2 \%$ of $\mathrm{BN}$ patients, $13.9 \%$ of EDNOS and $12.4 \%$ of AN individuals did not have breakfast before going to school $(P<0.0005)$. Finally, fast food restaurants were more frequently visited by EDNOS individuals $(17.6 \%)$ than by AN (13.9\%) and BN $(10.3 \%)$ patients $(P=0.004)$.

Table 2 includes the frequency distribution (means and standard deviations) of the derived CatPCA factor scores for ED patients (cases) and controls. In the total European sample, ANOVA comparisons adjusted by sex and age indicated that cases obtained higher means in the factors 'food used as individualization' $(P=0 \cdot 001)$, 'control and rules about food' $(P=0 \cdot 001)$ and 'food deprivation' $(P=0 \cdot 007)$, and lower means in the factor 'healthy eating' $(P=0.001)$; no differences between cases and controls were found for the factor 'food utilized as social glue'. Considering the country of origin, the first factor 'food used as individualization' obtained significant differences in the UK $(P=0.001)$ and Spain $(P=0 \cdot 003)$. The second factor 'control and rules about food' obtained higher means in ED patients in the UK $(P=0 \cdot 001)$, Slovenia $(P=0 \cdot 001)$ and Italy $(P=0.006)$. The third factor 'food utilized as social glue' differed significantly between ED cases and controls in Slovenia and Italy $(P=0.001$ in both samples). For the fourth factor, 'healthy eating', Austria $(P=0 \cdot 001)$ was the only country that was found to differ significantly between cases and controls. Finally, no significant differences between patients and controls were revealed for the fifth factor, 'Food deprivation'.

\section{Association of the derived categorical principal components analysis factors and the presence of an eating disorder diagnosis}

Table 3 contains the logistic models adjusted by sex and age that valued the predictive accuracy of the factor scores on the presence of an ED diagnosis. Considering the total European sample, higher scores in the factors 'food used as individualization' (OR 1.76) and 'control and rules about food' (OR 1.75) increased the likelihood of suffering from an ED. On the other hand, lower scores in the factor 'healthy eating' (OR 0.63) were negatively related to the development of a subsequent ED. This model obtained correct adjustment $(P=0.404)$ and moderate predictive accuracy $\left(R^{2} 0 \cdot 13\right.$; AUC 0.68).

In the UK, the possibility of an ED was increased with higher scores in the factors 'food used as individualization' (OR 1.70) and 'control and rules about food' (OR 1.97). In the Spanish sample, the only statistical association with an ED diagnosis was for high scores in the factor 'food used as individualization' (OR 2.04). On the other hand, scores in the factor 'healthy eating' diminished the probability of developing an ED in Austria (OR 0.32), and the factor 'food utilized as social glue' was negatively related to an ED diagnosis in Italy (OR 0.63). In Slovenia, an ED diagnosis was positively related to scores in the factor "control and rules

Table 2. Distribution for empirical factor scores in eating disorder cases and controls $†$

(Mean values and standard deviations)

\begin{tabular}{|c|c|c|c|c|c|c|c|c|c|c|c|c|c|}
\hline & & \multicolumn{2}{|c|}{ UK ( $n$ 503) } & \multicolumn{2}{|c|}{ Spain (n 422) } & \multicolumn{2}{|c|}{ Austria ( $n$ 153) } & \multicolumn{2}{|c|}{ Slovenia ( $n$ 294) } & \multicolumn{2}{|c|}{ Italy (n 292) } & \multicolumn{2}{|c|}{ Total $(n 1664)$} \\
\hline & & Mean & SD & Mean & SD & Mean & SD & Mean & SD & Mean & SD & Mean & SD \\
\hline \multirow[t]{3}{*}{ Factor 1} & Cases & 0.66 & 0.99 & 0.25 & 0.49 & 0.21 & 0.48 & 0.13 & 0.48 & 0.26 & 0.46 & 0.39 & 0.73 \\
\hline & Controls & 0.25 & 0.66 & 0.12 & 0.30 & 0.25 & 0.46 & 0.16 & 0.43 & 0.19 & 0.44 & 0.19 & 0.48 \\
\hline & $P$ value & $0.00^{\star *}$ & & $0.00^{* *}$ & & 0.64 & & 0.67 & & 0.19 & & $0.00^{\star *}$ & \\
\hline \multirow[t]{3}{*}{ Factor 2} & Cases & 0.51 & 0.61 & 0.04 & 0.55 & 0.00 & 0.47 & 0.31 & 0.61 & 0.48 & 0.60 & 0.30 & 0.62 \\
\hline & Controls & 0.25 & 0.57 & -0.03 & 0.51 & -0.02 & 0.55 & -0.04 & .046 & 0.30 & 0.50 & $0 \cdot 10$ & 0.05 \\
\hline & $P$ value & $0.00^{\star \star}$ & & 0.15 & & 0.79 & & $0.00^{\star \star}$ & & $0.01^{*}$ & & $0.00^{\star \star}$ & \\
\hline \multirow[t]{3}{*}{ Factor 3} & Cases & 0.00 & 0.99 & 0.48 & 0.86 & 0.27 & 0.92 & 0.40 & 0.94 & 0.38 & 0.88 & 0.26 & 0.94 \\
\hline & Controls & -0.07 & 0.95 & 0.46 & 0.80 & $0 \cdot 10$ & 0.98 & -0.06 & 0.94 & 0.74 & 0.71 & 0.21 & 0.93 \\
\hline & $P$ value & 0.45 & & 0.89 & & 0.28 & & $0.00^{\star *}$ & & $0.00^{\star *}$ & & 0.27 & \\
\hline \multirow[t]{3}{*}{ Factor 4} & Cases & $0 \cdot 18$ & $0 \cdot 70$ & -0.18 & 0.48 & $0 \cdot 10$ & 0.68 & 0.27 & 0.54 & -0.05 & 0.50 & 0.03 & 0.62 \\
\hline & Controls & 0.18 & 0.75 & -0.12 & 0.42 & 0.50 & 0.74 & 0.39 & 0.61 & -0.15 & 0.43 & 0.14 & 0.64 \\
\hline & $P$ value & 0.92 & & 0.23 & & $0.00^{\star *}$ & & 0.16 & & 0.07 & & $0.00^{\star \star}$ & \\
\hline \multirow[t]{3}{*}{ Factor 5} & Cases & 0.31 & 0.47 & -0.17 & 0.36 & 0.25 & 0.42 & 0.15 & 0.32 & 0.64 & 0.71 & 0.30 & 0.50 \\
\hline & Controls & 0.26 & 0.41 & 0.17 & 0.38 & $0 \cdot 14$ & 0.35 & 0.11 & 0.30 & 0.51 & 0.68 & 0.24 & 0.46 \\
\hline & $P$ value & 0.23 & & 0.84 & & 0.12 & & 0.36 & & 0.11 & & $0.01^{*}$ & \\
\hline
\end{tabular}

$P$ values are based on ANOVA procedures adjusted by sex and age; two-sided significance level: ${ }^{\star} P<0.05,{ }^{\star \star} P<0.01$

†For details of procedures and factors, see Methods and Table 1. 
about food' (OR 4.51). All the independent models for countries included in Table 3 obtained correct adjustment ( $P>0.05$ in Hosmer-Lemeshow's tests). The most predictive models corresponded to Slovenia $\left(R^{2} 0.42\right.$; AUC 0.88) and Austria $\left(R^{2} 0 \cdot 28\right.$; AUC 0.76).

Association of the derived categorical principal components analysis factors and the presence of an eating disorder subtype

Table 4 shows the multinomial logistic regression models adjusted by sex and age that valued the predictive accuracy of factor scores on the presence of a concrete ED subtype. The Slovenian sample was omitted in this analysis due the lack of information of the subtype diagnosis for these patients. In the total European sample, the factors increasing the likelihood of any subtype (AN, BN and EDNOS) were 'food used as individualization' and 'control and rules about food', and the factor negatively related to all ED subtypes was 'healthy eating'. This model obtained moderate predictive accuracy $\left(R^{2} 0 \cdot 136\right)$.

Considering the country of origin, 'food used as individualization' increased the incidence of all ED subtypes in the UK. In Spain, this factor was also positively related to the development of AN and BN. The factor 'control and rules about food' augmented the probability of presenting $\mathrm{AN}$ and $\mathrm{BN}$ in the UK and of developing BN in Italy. Conversely, 'food utilized as social glue' decreased the likelihood of developing $\mathrm{AN}$ in Italy. The factor 'healthy eating' diminished the occurrence of AN in Spain, and it was also negatively related to AN and EDNOS in Austria. On the contrary, in Italy 'healthy eating' increased the probability of developing BN. Finally, the factor 'food deprivation' was negatively related to AN in Spain. The predictive accuracy of the concrete models for each country was moderate, and ranged between $R^{2} 0.096$ for Spain to $R^{2} 0.372$ for Austria.

\section{Discussion}

The instrument that was employed in the present study seems to be sensitive to the food-related environmental factors that might be related to the development of a subsequent ED. In the total sample we found that the five domains established through the CatPCA were of relevance although most of these were of small effects. In accordance with our first hypothesis, we found that the domains 'food used as individualization' and 'control and rules about food' increased the probability of developing a later ED. Contrastingly, 'healthy eating' was found to be negatively associated with a subsequent ED. Furthermore, as hypothesized, some differences across countries were reported. Finally, in relation to our third and fourth aim only a few differences were observed for the derived CatPCA dimensions across ED sub-diagnoses, the total European sample and the distinct countries. The present unexpected results could to some extent have resulted from the low predictive capacity reported in most of the assessed models.

\section{Food as individualization}

In relation to our first aim, which examined whether some of the early patterns of eating behaviour were related to the development of a subsequent ED, we revealed that 'food 
Table 4. Predictive accuracy of empirical factor scores on eating disorder subtypes $†$

(Odds ratios and $95 \%$ confidence intervals)

\begin{tabular}{|c|c|c|c|c|c|c|c|c|c|c|}
\hline & & \multicolumn{3}{|c|}{ Anorexia } & \multicolumn{3}{|c|}{ Bulimia } & \multicolumn{3}{|c|}{ EDNOS } \\
\hline & & $P$ & OR & $95 \% \mathrm{Cl}$ & $P$ & OR & $95 \% \mathrm{Cl}$ & $P$ & OR & $95 \% \mathrm{Cl}$ \\
\hline \multirow[t]{5}{*}{ UK ( $\left.n 503 ; R^{2} 0.132\right)$} & Factor 1 & $0.02^{*}$ & 1.46 & $1.06,2.00$ & $0.01^{*}$ & 1.67 & $1 \cdot 12,2 \cdot 48$ & $0.00^{\star *}$ & $2 \cdot 00$ & $1.47,2.72$ \\
\hline & Factor 2 & $0.00^{* *}$ & 2.08 & $1 \cdot 38,3 \cdot 13$ & $0.00^{* \star}$ & 3.58 & $1.97,6.53$ & $0.07^{\star}$ & 1.50 & $0.97,2.31$ \\
\hline & Factor 3 & 0.73 & 1.04 & $0.82,1.33$ & 0.64 & 1.09 & $0.75,1.58$ & 0.95 & 1.01 & $0.78,1.30$ \\
\hline & Factor 4 & 0.12 & 0.77 & $0.55,1.08$ & 0.37 & 0.80 & $0.48,1.31$ & 0.54 & 0.90 & $0.63,1.27$ \\
\hline & Factor 5 & 0.79 & 1.08 & $0.61,1.92$ & 0.95 & 0.97 & $0.42,2.24$ & 0.36 & 1.31 & $0.73,2.32$ \\
\hline \multirow[t]{5}{*}{ Spain $\left(n 422 ; R^{2} 0.096\right)$} & Factor 1 & $0.01^{*}$ & 2.55 & $1.29,5.04$ & $0.05^{\star}$ & 1.92 & $1.01,3.65$ & 0.17 & 1.69 & $0.80,3.60$ \\
\hline & Factor 2 & 0.80 & 1.08 & $0.61,1.92$ & 0.57 & $1 \cdot 15$ & $0.71,1.88$ & 0.31 & 1.34 & $0.76,2.34$ \\
\hline & Factor 3 & 0.95 & 1.00 & $0.71,1.39$ & 0.95 & 0.99 & $0.73,1.34$ & 0.39 & $1 \cdot 18$ & $0.81,1.70$ \\
\hline & Factor 4 & $0.03^{*}$ & 0.45 & $0.22,0.92$ & 0.73 & 0.91 & $0.52,1.58$ & 0.31 & 0.70 & $0.35,1.39$ \\
\hline & Factor 5 & $0.03^{*}$ & 0.27 & $0.08,0.89$ & 0.40 & $1 \cdot 32$ & $0.69,2.56$ & 0.93 & 1.04 & $0.45,2.36$ \\
\hline \multirow[t]{5}{*}{ Austria $\left(n 153 ; R^{2} 0.372\right)$} & Factor 1 & 0.80 & $1 \cdot 17$ & $0.33,4.11$ & 0.45 & 0.41 & $0.04,4.18$ & 0.15 & $2 \cdot 39$ & $0.74,7.76$ \\
\hline & Factor 2 & 0.79 & 0.83 & $0.21,3.26$ & 0.44 & 1.64 & $0.46,5.78$ & 0.64 & 1.34 & $0.40,4.48$ \\
\hline & Factor 3 & 0.66 & $1 \cdot 16$ & $0 \cdot 60,2 \cdot 23$ & 0.17 & 1.83 & $0.78,4.29$ & 0.66 & $1 \cdot 17$ & $0.58,2.39$ \\
\hline & Factor 4 & $0.02^{*}$ & 0.29 & $0.10,0.82$ & $0.08^{*}$ & 0.41 & $0.15,1.13$ & $0.01^{*}$ & 0.24 & $0.09,0.65$ \\
\hline & Factor 5 & 0.72 & $1 \cdot 38$ & $0.24,8.04$ & 0.50 & $1 \cdot 81$ & $0.32,10.2$ & $0.07^{*}$ & 3.97 & $0.91,17.3$ \\
\hline \multirow[t]{5}{*}{ Italy ( $n$ 292; $\left.R^{2} 0 \cdot 186\right)$} & Factor 1 & $0.06^{*}$ & $1 \cdot 79$ & $0.97,3.30$ & 0.15 & 0.50 & $0.20,1.28$ & 0.35 & 1.92 & $0.50,7.40$ \\
\hline & Factor 2 & 0.50 & $1 \cdot 21$ & $0.69,2.13$ & $0.03^{\star}$ & 1.90 & $1.07,3.33$ & 0.40 & 0.55 & $0.13,2.26$ \\
\hline & Factor 3 & $0.00^{\star *}$ & 0.55 & $0.37,0.80$ & 0.12 & 0.71 & $0.47,1.09$ & 0.32 & 0.67 & $0.31,1.46$ \\
\hline & Factor 4 & 0.68 & 0.87 & $0.44,1.69$ & $0.03^{*}$ & $2 \cdot 11$ & $1.07,4.16$ & 0.51 & 0.59 & $0.12,2.84$ \\
\hline & Factor 5 & 0.77 & 0.94 & $0.59,1.48$ & 0.42 & 1.22 & $0.75,2.00$ & 0.28 & 1.62 & $0.67,3.90$ \\
\hline \multirow{5}{*}{$\begin{array}{l}\text { European sample } \\
\qquad\left(n 1370 ; R^{2} 0 \cdot 136\right)\end{array}$} & Factor 1 & $0.00^{*}$ & 1.81 & $1 \cdot 44,2 \cdot 28$ & $0.01^{*}$ & 1.45 & $1.11,1.90$ & $0.00^{\star *}$ & $2 \cdot 34$ & $1 \cdot 85,2 \cdot 97$ \\
\hline & Factor 2 & $0.00^{\star *}$ & 1.82 & $1 \cdot 42,2.33$ & $0.00^{\star \star}$ & 1.86 & $1.42,2.43$ & $0.01^{*}$ & 1.48 & $1.12,1.97$ \\
\hline & Factor 3 & 0.58 & 0.96 & $0.83,1.11$ & 0.11 & $1 \cdot 15$ & $0.97,1.37$ & 0.84 & 0.98 & $0.83,1.17$ \\
\hline & Factor 4 & $0.00^{\star \star}$ & 0.51 & $0.41,0.66$ & $0.00^{\star \star}$ & 0.62 & $0.48,0.81$ & $0.00^{*}$ & 0.66 & $0.51,0.86$ \\
\hline & Factor 5 & 0.77 & 0.96 & $0.71,1.29$ & 0.16 & 1.25 & $0.92,1.70$ & 0.88 & 1.03 & $0.74,1.43$ \\
\hline
\end{tabular}

EDNOS, eating disorders not otherwise specified; $R^{2}$, Nagelkerke's coefficient.

Multinomial regressions (ENTER procedure) adjusted by sex and age (reference category: control group); two-sided significance level: ${ }^{\star} P<0.05$, ${ }^{\star \star} P<0.01$.

†For details of procedures and factors, see Methods and Table 1. Data from Slovenia are not included due the lack of diagnose subtype in this sample.

used as individualization' was positively associated with a later ED. The need for individualized foods within the family (father, siblings and own patients) might be related to the increased risk of ED within the family by decreasing social bonding. However, it could also be a marker of multiple domains, and could measure the importance of food for individualization and perhaps respect within the family or it could also be a measure of picky eating as shown in previous studies $^{(10,12)}$. In accordance with the present findings, previous research has indicated that adolescents who struggled for autonomy, disliked food served at family meals and often refused to attend family meals ${ }^{(16,46)}$. However, other studies have documented that regardless of their longing for independence and experimentation, adolescents ultimately slipped back to favour the same foods as their parents ${ }^{(47)}$. More demanding lifestyles, such as extended working and school hours, increased after-school activities, transformations in family and living arrangements, and augmented accessibility of convenience and fast foods could be responsible for an increased right to be special or different about food and its environment $^{(48,49)}$.

\section{Control and rules about food}

Another domain from the CatPCA that was found to increase the probability of presenting a later ED in the whole European sample was 'control and rules about food'. Previous studies have suggested that parents who employ food control strategies (e.g. reward, punishment or limiting access to certain foods) may actually discourage the formation of adequate control over eating in their children ${ }^{(50-52)}$. Also, research has shown that limiting access to food as a form of punishment was associated with an increased incidence of $\mathrm{BN}$ and binge ED. One hypothesis is that parents might restrict access to foods in response to their child's adiposity or snacking behaviour ${ }^{(19-50)}$. A second hypothesis is that mothers who themselves have eating problems may project their own control and rules about eating on to their offspring ${ }^{(10,20)}$. Only further longitudinal research will reveal the potentional mediating role of parental rules and control about food in the aetiological factors and clinical course of ED.

\section{Healthy eating}

The domain of 'healthy eating'(especially mother, father and own patient paid attention to healthy eating) from the CatPCA appeared to be negatively related to a later ED. In agreement with the present results, previous studies have also demonstrated that parents' modelling of optimal dietary behaviours was associated with children's intake of healthy foods and favourable eating patterns ${ }^{(53-55)}$. Healthy eating has also often been related to structured family meals, a high priority for family meals and an encouraging environment at family meals ${ }^{(56-61)}$, and may contribute to a positive psychosocial development in children and adolescents ${ }^{58,62,63)}$.

\section{Differences across countries in early eating behaviour}

The secondary aim of the present study was to examine whether there were differences across countries in these 
early eating behaviours. The pattern of associated ED factors did vary between countries. In the UK and Spain, 'food used as individualization' was positively related to a later ED; 'control and rules about food' increased the incident of ED in the UK and Slovenia. In Austria 'healthy eating' was negatively related to an ED diagnosis and in Italy 'food utilized as social glue' diminished the incidence of ED.

Disparities in dietary habits across European populations may be related to different culutral norms, socio-economic differences, unstable political situations and variations in level of education achieved $^{(64,65)}$. However, it should also be noted that even though there appear to be 'traditional cultures of eating habits' across EU countries, globally there are modern ones, such as 'fast food restaurants' which have started to enter our everyday life. Plotting such information on a timescale, documenting stable habitual eating habits $v$. new ones, would therefore be very important for nutrition research ${ }^{(66)}$

Our final two aims were to assess whether there were differences across ED sub-diagnoses for the total European sample and the distinct countries. In contrast to our expectations, we did not reveal differences in the assessed eating behaviours across ED sub-diagnoses. Similarly, when the distinct countries were assessed independently also only a few differences between ED sub-diagnoses emerged, which partially could be attributable to low statistical power.

\section{Limitations and strengths of the present study}

The present study has several limitations that need to be highlighted. The major limitation was that the instrument was retrospective. Therefore the risk and protective factors that we have defined are no more than retrospective correlates according to the criteria of Kraemer et al. ${ }^{(67)}$. Therefore it will be important to confirm the relevance of these factors in prospective studies. Furthermore, the case mix including a high proportion of clinical cases may have limited the validity and the reliability of the present findings. Also even though the detail about food and eating defined in this instrument was greater than in previous longitudinal studies, further definition and clarification is probably needed. For example, the meaning in translation of the value of food may lack precision. This may explain the lack of consistency in the link between ED and this item between countries. Nevertheless within a specific culture the meanings may be clear as exemplified by the high levels of test-retest reliability reported in a previous study ${ }^{(8)}$. Furthermore, it should be mentioned that feeding style is not just an environmental measure. Gene environment correlation highlights that the same individuals who provide our environment also provide us with our genes. Hence, some of the feeding practices seen in this paper could be correlated with genetically mediated characteristics of the parents that were unmeasured (e.g. parental ED, parental BMI). Finally, the sample sizes (for ED subtypes and control cohorts) and the success of the matching between controls and patients varied between the different countries, which affected the accuracy of the $P$ values and the estimation in the regression models.

Notwithstanding these limitations, this is a large sample which has addressed early eating behaviours and the role of family values and attitudes in a standard way between different cultures in Europe. No previous study has relied on such a large sample. ED are low prevalence disorders, therefore any study aimed at clarifying risk factors for ED requires a large enough study. We were able to determine whether associated ED factors varied in each of the recruiting countries, due to the large sample size across the countries.

The findings from the present study may help to shape appropriate prevention and early intervention programmes, and identify 'at risk' (children and adolescent) individuals. The current findings indicate that nutritional policies and education should take into consideration that food and eating are influential manifestations of cultural and social identities and that these vary considerably across Europe. Such prevention efforts should include a variety of environmental factors such as informing the family to provide food within a structured context, to pay attention and monitor the youngster's eating patterns and to role-model healthy-eating practices ${ }^{(68,69)}$.

The present study enhances our knowledge about the association between ED and unhealthy eating patterns in childhood and early adolescence, but several unanswered questions remain for future studies. Future research could expand the present results by employing longitudinal designs addressing the potential mediating role of individual and family eating patterns in the aetiological factors and clinical course of ED. Moreover, forthcoming research could examine whether the people with a particular genotype and common psychosocial factors (as those studied here) are more vulnerable for developing an ED and to determine how various risk factors interact in increasing the risk for disordered eating.

In conclusion, the present study focused on a variety of environmental and social eating-related factors in a large case control sample from various European countries, which are potentially modifiable early markers for ED. The findings from the present study agree with the growing body of research indicating that dysfunctional individual and family eating patterns in childhood and early adolescence could lead to the development of a subsequent ED. The present findings will therefore be imperative for developing models of risk using similar designs of populations from across Europe.

\section{Acknowledgements}

Financial support was received from the European Union (Framework-V Multicenter Research Grant, QCK1-1999-916), Fondo de Investigación Sanitaria (CIBER; CB06/03/0034), Generalitat de Catalunya (2005SGR00322), FI (2005 FI 00425) and BE (100172). The CIBER Fisiopatologia de la obesidad y Nutricion is an initiative of ISCIII. This work is part of the $\mathrm{PhD}$ dissertation of I. K. at the University of Barcelona. There is no conflict of interest. All authors designed the study, wrote the protocol and managed the literature searches and summaries of previous related work. R. G. and E. P. undertook the statistical analysis, and and I. K., J. T. and F. F.-A. wrote wrote the first draft of the manuscript. All authors contributed to and have approved the final manuscript.

\section{References}

1. Jacobi C, Hayward C, de Zwaan M, et al. (2004) Coming to terms with risk factors for eating disorders: application of risk terminology and suggestions for a general taxonomy. Psychol Bull 130, 19-65. 
2. Karwautz A, Rabe-Hesketh S, Hu X, et al. (2001) Individualspecific risk factors for anorexia nervosa: a pilot study using a discordant sister-pair design. Psychol Med 31, 317-329.

3. Steiger H \& Bruce KR (2007) Phenotypes, endophenotypes, and genotypes in bulimia spectrum eating disorders. Can J Psychiatry 52, 220-227.

4. Treasure JL (2007) Getting beneath the phenotype of anorexia nervosa: the search for viable endophenotypes and genotypes. Can J Psychiatry 52, 212-219.

5. Calam R \& Waller G (1998) Are eating and psychosocial characteristics in early teenage years useful predictors of eating characteristics in early adulthood? A 7-year longitudinal study. Int J Eat Disord 24, 351-362.

6. Fairburn CG, Cooper Z, Doll HA, et al. (1999) Risk factors for anorexia nervosa: three integrated case-control comparisons. Arch Gen Psychiatry 56, 468-476.

7. Stice E \& Whitenton K (2002) Risk factors for body dissatisfaction in adolescent girls: a longitudinal investigation. Dev Psychol 38, 669-678.

8. Fernández-Aranda F, Krug I, Granero R, et al. (2007) Individual and family eating patterns during childhood and early adolescence: an analysis of associated eating disorder factors. Appetite 49, 476-485.

9. Jacobi C, Agras WS, Bryson S, et al. (2003) Behavioural validation, precursors, and concomitants of picky eating in childhood. J Am Acad Child Adolesc Psychiatry 42, 76-84.

10. Micali N, Holliday J, Karwautz A, et al. (2007) Childhood eating and weight in eating disorders: a multi-centre European study of affected women and their unaffected sisters. Psychother Psychosom 76, 234-241.

11. Kortegaard LS, Hoerder K, Joergensen J, et al. (2001) A preliminary population-based twin study of self-reported eating disorder. Psychol Med 31, 361-365.

12. Marchi M \& Cohen P (1990) Early childhood eating behaviours and adolescent eating disorders. J Am Acad Child Adolesc Psychiatry 29, 112-117.

13. Kotler LA, Cohen P, Davies M, et al. (2001) Longitudinal relationships between childhood, adolescent, and adult eating disorders. J Am Acad Child Adolesc Psychiatry 40, 1434-1440.

14. Gillberg IC, Gillberg C, Rastam M, et al. (1996) The cognitive profile of anorexia nervosa: a comparative study including a community-based sample. Compr Psychiatry 37, 23-30.

15. Rastam M \& Gillberg C (1996) Anorexia nervosa rates - conclusions for the wrong reasons. Br J Psychiatry 168, 251-252.

16. Birch LL \& Fisher JO (1998) Development of eating behaviours among children and adolescents. Pediatrics 101, $539-549$

17. Birch LL, Fisher JO, Grimm-Thomas K, et al. (2001) Confirmatory factor analysis of the child feeding questionnaire: a measure of parental attitudes, beliefs and practices about child feeding and obesity proneness. Appetite 36, 201-210.

18. Dick DM, Johnson JK, Viken RJ, et al. (2000) Testing betweenfamily associations in within-family comparisons. Psychol Sci 11, 409-413.

19. Faith MS \& Kerns J (2005) Infant and child feeding practices and childhood overweight: the role of restriction. Matern Child Nutr 1, 164-168.

20. Edmunds H \& Hill AJ (1999) Dieting and the family context of eating in young adolescent children. Int $J$ Eat Disord 25, $435-440$.

21. Fairburn CG, Welch SL, Doll HA, et al. (1997) Risk factors for bulimia nervosa. A community-based case-control study. Arch Gen Psychiatry 54, 509-517.

22. Taylor CB, Bryson S, Celio Doyle AA, et al. (2006) The adverse effect of negative comments about weight and shape from family and siblings on women at high risk for eating disorders. Pediatrics 118, 731-738.
23. Cullen KW, Baranowski T, Rittenberry L, et al. (2000) Socialenvironmental influences on children's diets: results from focus groups with African-Euro- and Mexican American children and their parents. Health Educ Res 15, 581-590.

24. Nakamura K, Hoshino Y, Watanabe A, et al. (1999) Eating problems and related weight control behaviour in adult Japanese women. Psychother Psychosom 68, 51-55.

25. Soh NL, Touyz SW \& Surgenor LJ (2006) Eating and body image disturbances across cultures: a review. Eur Eat Disord $\operatorname{Rev~14,~54-65.~}$

26. Keel PK \& Klump KL (2003) Are eating disorders culture-bound syndromes? Implications for conceptualizing their aetiology. Psychol Bull 129, 747-769.

27. Miller MN \& Pumariega AJ (2001) Culture and eating disorders: a historical and cross-cultural review. Psychiatry 64, 93-110.

28. Furnham A \& Adam-Saib S (2001) Abnormal eating attitudes and behaviours and perceived parental control: a study of white British and British Asian school girls. Soc Psychiatry Psychiatr Epidemiol 36, 462-470.

29. Hoste RR, Hewell K \& le Grange D (2007) Family interaction among white and ethnic minority adolescents with bulimia nervosa and their parents. Eur Eat Disord Rev 15, 152-158.

30. Striegel-Moore RH, Wilfley DE, Pike KM, et al. (2000) Recurrent binge eating in black American women. Arch Fam Med 9, $83-87$.

31. Striegel-Moore RH \& Bulik CM (2007) Risk factors for eating disorders. Am Psychol 62, 181-198.

32. Buddeberg-Fischer B, Bernet R, Schmid J, et al. (1996) Relationship between disturbed eating behaviour and other psychosomatic symptoms in adolescents. Psychother Psychosom 65, 319-326.

33. Faravelli C, Ravaldi C, Truglia E, et al. (2006) Clinical epidemiology of eating disorders: results from the Sesto Fiorentino study. Psychother Psychosom 75, 376-383.

34. Santonastaso P, Zanetti T, Sala A, et al. (1996) Prevalence of eating disorders in Italy: a survey on a sample of 16-year-old female students. Psychother Psychosom 65, 158-162.

35. American Psychiatric Association (2000) American Psychiatric Association Diagnostic and Statistical Manual of Mental Disorders, 4th ed., revised. Washington, DC: American Psychiatric Association.

36. First M, Gibbon M, Spitzer R, et al.VersionUsers Guide for the Structured Clinical Interview for DSM IV Axis I Disorders Research Version (SCID-I, Version 2.0). New York: New York State Psychiatric Institute.

37. Goldberg DP (1981) Manual of the General Health Questionnaire (GHQ-28). Toronto: NFER Nelson Publishing.

38. Garner DM, Olmsted MP, Bohr Y, et al. (1982) The eating attitudes test: psychometric features and clinical correlates. Psychol Med 12, 871-878.

39. Fairburn CG, Doll HA, Welch SL, et al. (1998) Risk factors for binge eating disorder: a community-based, case-control study. Arch Gen Psychiatry 55, 425-432.

40. Shisslak CM, Renger R, Sharpe T, et al. (1999) Development and evaluation of the McKnight risk factor survey for assessing potential risk and protective factors for disordered eating in preadolescent and adolescent girls. Int $J$ Eat Disord 25, 195-214.

41. Bonillo A, Granero R \& Krug I (2006) Psychometric reliability and validity of the cross-cultural (environmental) questionnaire (CCQ) Poster presented at the Hispano Latinoamericano Congress on Eating Disorders, 6 June, Barcelona, Spain.

42. Keller MB, Lavori PW, Friedman B, et al. (1987) The longitudinal interval follow-up evaluation. A comprehensive method for assessing outcome in prospective longitudinal studies. Arch Gen Psychiatry 44, 540-548. 
43. Fairburn CG \& Cooper Z (1993) The Eating Disorder Examination (12 th Ed.). Binge Eating: Nature, Assessment and Treatment, pp. 317-360 [CG Fairburn and GT Wilson, editors]. New York: Guilford Press.

44. Goldberg D \& Williams P (1996) Cuestionario de salud general GHQ (General health questionnaire). Guia para el usuario de las distintas versiones, Barcelona.

45. Steiner H \& Lock J (1998) Anorexia nervosa and bulimia nervosa in children and adolescents: a review of the past 10 years. J Am Acad Child Adolesc Psychiatry 37, 352-359.

46. Neumark-Sztainer D, Story M, Dixon LB, et al. (1998) Adolescents engaging in unhealthy weight control behaviours: are they at risk for other health-compromising behaviours? Am J Public Health 88, 952-955.

47. Branen L, Fletcher J \& Hilbert L (2002) Snack consumption and waste by preschool children served 'Cute' versus regular snacks. J Nutr Educ Behav 34, 279-282.

48. Neumark-Sztainer D, Story M, Hannan PJ, et al. (2002) Weight-related concerns and behaviours among overweight and non-overweight adolescents: implications for preventing weight-related disorders. Arch Pediatr Adolesc Med 156, 171-178.

49. Neumark-Sztainer D, Story M, Hannan PJ, et al. (2002) Overweight status and eating patterns among adolescents: where do youths stand in comparison with the healthy people 2010 objectives? Am J Public Health 92, 844-851.

50. Birch LL \& Fisher JO (2000) Mothers' child-feeding practices influence daughters' eating and weight. Am J Clin Nutr 71, $1054-1061$.

51. Ogden J, Reynolds R \& Smith A (2006) Expanding the concept of parental control: a role for overt and covert control in children's snacking behaviour? Appetite 47, 100-106.

52. Fisher JO \& Birch LL (1999) Restricting access to palatable foods affects children's behavioural response, food selection, and intake. Am J Clin Nutr 69, 1264-1272.

53. Birch LL \& Lee Y (2002) Family influences: mothers' and daughters' use of multivitamin mineral supplements. Nutr Today 37, 173-174.

54. Hayman LL (2003) The dietary intervention study in children (disc): progress and prospects for primary prevention. Prog Cardiovasc Nurs 18, 4-5.

55. Patrick H \& Nicklas TA (2005) A review of family and social determinants of children's eating patterns and diet quality. J Am Coll Nutr 24, 83-92.

56. Fulkerson JA, Neumark-Sztainer D \& Story M (2006) Adolescent and parent views of family meals. J Am Diet Assoc 106, 526-532.
57. Fulkerson JA, Story M, Mellin A, et al. (2006) Family dinner meal frequency and adolescent development: relationships with developmental assets and high-risk behaviours. J Adolesc Health 39, 337-345.

58. Neumark-Sztainer D, Wall M, Story M, et al. (2004) Are family meal patterns associated with disordered eating behaviours among adolescents? J Adolesc Health 35, 350-359.

59. Sjoberg A, Hallberg L, Hoglund D, et al. (2003) Meal pattern, food choice, nutrient intake and lifestyle factors in the Goteborg adolescence study. Eur J Clin Nutr 57, 1569-1578.

60. Videon TM \& Manning CK (2003) Influences on adolescent eating patterns: the importance of family meals. J Adolesc Health 32, 365-373.

61. Worobey J (2002) Early family mealtime experiences and eating attitudes in normal weight, underweight and overweight females. Eat Weight Disord 7, 39-44.

62. Neumark-Sztainer D, Story M, Hannan PJ, et al. (2000) Disordered eating among adolescents: associations with sexual/ physical abuse and other familial/psychosocial factors. Int $J$ Eat Disord 28, 249-258.

63. Gillman MW, Rifas-Shiman SL, Frazier AL, et al. (2000) Family dinner and diet quality among older children and adolescents. Arch Fam Med 9, 235-240.

64. Dowler E (2001) Inequalities in diet and physical activity in Europe. Public Health Nutr 4, 701-709.

65. Irala-Estevez JD, Groth M, Johansson L, et al. (2000) A systematic review of socio-economic differences in food habits in Europe: consumption of fruit and vegetables. Eur J Clin Nutr 54, 706-714.

66. Oltersdorf U, Schlettwein-Gsell D \& Winkler G (1999) Assessing eating patterns - an emerging research topic in nutritional sciences: introduction to the symposium. Appetite $32,1-7$.

67. Kraemer HC, Stice E, Kazdin A, et al. (2001) How do risk factors work together? Mediators, moderators, and independent, overlapping, and proxy risk factors. Am J Psychiatry 158, $848-856$.

68. Bryant-Waugh R, Turner H, East P, et al. (2007) Developing a parenting skills-and-support intervention for mothers with eating disorders and pre-school children part 1: qualitative investigation of issues to include. Eur Eat Disord Rev 15, 350-356.

69. Bryant-Waugh R, Turner H, Jones C, et al. (2007) Developing a parenting skills-and-support intervention for mothers with eating disorders and pre-school children: part 2. Piloting a group intervention. Eur Eat Disord Rev 15, 439-448. 